\title{
Role of Magnetic Resonance Imaging in Spinal Cord Traumatic Injuries and its Outcome according to ASIA Impairment Scale
}

\author{
Tanweer Ahmad, ${ }^{1}$ Omer Sajjad, ${ }^{1}$ Mian Waheed Ahmad ${ }^{1}$
}

\begin{abstract}
Background: Spinal trauma is one of common neurosurgical problem and mismanagement can lead to fatal outcome. Objective: To determine the MRI based initial diagnosis of acute spinal injuries and determine its pattern with reference to its neurological outcome.

Methodology: Study Design: Prospective cohort study, involving radiographic and clinical review. This study was performed on 69 patients of spinal trauma, January to December, 2018, at Department of Radiology, Gujranwala Medical College and DHQ teaching Hospital, Gujranwala. Male and female of any age were included. After initial neurological examination these patients undergone for Magnetic Resonance Imaging of spine, which were analyzed and correlated with American Spinal Injury Association (ASIA) impairment scale (AIS), then subsequently at sub-acute interval to assess neurological outcome. Data analysis was done on SPSS version 17.

Results: The most common MRI findings were spinal cord edema 24 cases (34.7\%). Among the other findings were, cord contusion 17 cases $(24.6 \%)$, spinal cord deformity due to extrinsic compression 14 cases $(20.2 \%)$, focus of hemorrhage within the cord 9 cases (13\%), epidural hematoma 3 cases $(4.3 \%)$ and few cases appeared normal cord 2 cases $(2.3 \%)$. Most of the MRI findings correlated with clinical presentation of the patient according to American Spinal Injury Association (ASIA) impairment scale.

Conclusion: Patients with initial hemorrhagic grade had very poor prognosis, whereas those with focal spinal cord edema had good clinical recovery.

Keywords: Magnetic Resonance Imaging, Spinal cord injury, American Spinal Injury Association, Gradient - echo, Spinal cord edema, Cord contusion
\end{abstract}

\section{Introduction}

Spinal cord injury (SCI) means any insult of spinal cord resulting in a change, either permanent or temporary, in the cord's normal sensory, motor or autonomic functions. ${ }^{1}$ Persons with spinal cord injuries, usually have permanent and often devastating neurologic deficits and disability.1 As compared with different other disabling conditions, spinal cord injury (SCI) relatively affects small number of people; each year about 11,000 peoples are added. Current United States total of approximately 230,000 people with this condition.

Trauma to the spinal cord and spine is probably devastating injury. It can be associated with vital neurological insult including quadriplegia, paraplegia or even death. The patients on first presentation with severe cord injury with intact sensory motor function on initial neurological examination have very poor prognosis. ${ }^{2}$ On the other hand, patients who present with an incomplete injury may regain a large amount of useful function or be spared, the progression to complete injury with rapid diagnosis and treatment of fracture fragments, hematomas or other lesions, which compress the spinal cord. Imaging studies are essential to confirm the exact location of the injury, to assess the stability of spine, and to redefine the repercussion of the trauma on the diameters of the spinal canal and neural foramina as well as on the spinal cord and nerve roots. It has been shown that MRI characterized three MRI signal patterns for the prognosis of acute spinal cord injury (SCI): hemorrhage in the spinal cord; focal edema or myelitis of the spinal cord; and combination of hemorrhage and edema. ${ }^{3}$

Magnetic Resonance Imaging of the spinal cord of traumatized person describes detail anatomic structures and it can help to determine the extent of the probable mechanism of spinal injury, degree of neurological deficit and the presence of spinal instability. One of the key reason that limits, why MRI spine is not used frequently in acutely traumatized cases, is the logistics of injured transport and monitoring. 5 Neurologist and neurosurgeons are also confronted with multitude of available MRI sequences. Appropriate selection of MRI sequences for diagnosis and prognosis can simplify imaging with in limiting costs. ${ }^{6,7}$ Further sub classification of injury defined by the American Spinal Injury Association (ASIA) Impairment Scale (modified

1. Department of Radiology, Gujranwala Medical College and DHQ teaching Hospital, Gujranwala

Correspondence: Tanweer Ahmad, Department of Radiology, Gujranwala Medical College and DHQ teaching Hospital, Gujranwala 
from the Frankel classification), using the following categories: $8 \mathrm{~A}=$ Complete: No sensory or motor function is preserved in sacral segments S4-S5; B = Incomplete: Sensory, but not motor, function is preserved below the neurologic level and extends through sacral segments S4-S5; C = Incomplete: Motor function is preserved below the neurologic level, and most key muscles below the neurologic level have a muscle grade of less than 3; $\mathrm{D}=$ Incomplete: Motor function is preserved below the neurologic level, and most key muscles below the neurologic level have a muscle grade that is greater than or equal to $3 ; \mathrm{E}=$ Normal: Sensory and motor functions are normal. The objective of this study was to determine the MRI based initial diagnosis of acute spinal injuries and determine its pattern with reference to its neurological outcome.

\section{Methodology}

This prospective study was conducted over a period of about one year from $1^{\text {st }}$ January to $31^{\text {st }}$ December, 2018, at Department of Radiology, Gujranwala Medical College and DHQ teaching Hospital, Gujranwala. Patients with acute traumatic spinal injuries were included in the study. Data collection of the 69 patients including age, sex, date of trauma and sensory motor examination was carried out. All patients underwent MRI examination of specific region or whole spine according to requirement. Exclusion criteria include; patient with at least one absolute contraindication, non-cooperative patient, patients with metallic implants, claustrophobia, pacemakers, and cochlear implants in situ.

Acute spinal trauma patient required special consideration before imaging. All potential risks for imaging included medically unstable patient were carefully weighed against the need for the diagnostic information provided by MRI. Cervical spine injured patients were usually stabilized with fiberglass cervical collar. Sedation was used where necessary to complete the examination. Choice of surface coil was determined by the location(s) of injury, access to the area of interest, and the types of coils available. Study was conducted after ethical committee permission and data collection performed after informed consent. Data analysis was done by SPSS software version 17.

MRI techniques in spinal trauma: Study was conducted on Syngo SEIMENS 1.5T MR scanner. Sequences include axial and sagittal planes using a combination of pulse sequences. This study was performed with patient in supine position with quiet breathing obtaining axial T2 and T1-weighted fast spin echo images, STIR images and sagittal T2, T1weighted fast spin echo images. Sagittal images were $5.0 \mathrm{~mm}$ thick with a $0.5 \mathrm{~mm}$ slice gap. The field of view (FOV) of the area of interest is adequate at 22 $\mathrm{cm}$ in cervical spine and at $30 \mathrm{~cm}$ in lumbosacral spine. In the dorso-lumbar spine, a large FOV was needed $(34 / 36 \mathrm{~cm})$ for accurate labeling of the involved levels. Axial images were obtained using fast spine echo (FSE) or gradient-echo (GRE) pulse sequences. Technical parameters included low TR/TE, $16^{\circ}$ flip angle, $228 \times 320$ matrix and two excitations in T1WI and one excitation in T2WIs. The TE used was less than $15 \mathrm{~ms}$ in T1WI and up to $100 \mathrm{~ms}$ in T2WIs in order to minimize unwanted susceptibility effects that might exaggerate bony stenosis.

\section{Results}

In this study, the mean age of patient was $31.55 \pm 19.13$ years. There were $51(73.9 \%)$ males and $18(26.1 \%)$ females presented for MRI diagnosis. The most common MRI finding was spinal cord edema that was observed in 24 (34.7\%) cases. Among the other findings were focus of cord contusion, which was observed in $17(24.6 \%)$ cases, spinal cord deformity due to extrinsic compression was observed in $14(20.2 \%)$ cases, focus of hemorrhage in 9 cases(13\%), epidural hematoma in 3 cases $(4.3 \%)$ while $2(2.3 \%)$ cases had normal spinal cordwithout neural compression, however, 6 cases had oblique fracture of D7 vertebra and 6 had fracture of L5 vertebral superior endplate at its anterior end but their neural structure was normal and no deformity was observed in spinal cord. Most of MR findings matched well with clinical profile of the injured patient according to ASIA impairment scale.

Person with severe injury including large focus of hemorrhage and extensive cord edema on initial ASIA impairment scale (AIS) showed poor recovery at follow up. Severe spinal cord compression was also associated with poor neurological outcome on follow up; however it was not statistically significant. Following data is shown in table I below. 
Table I: Comparison of ASIA impairment scale at the time of admission and discharge, in patients of spinal trauma

\begin{tabular}{|c|c|c|c|c|c|c|c|c|c|c|}
\hline \multirow{2}{*}{ MRI findings } & \multicolumn{5}{|c|}{$\begin{array}{l}\text { Number of patients at } \\
\text { Admission in each category }\end{array}$} & \multicolumn{5}{|c|}{$\begin{array}{c}\text { Number of patients at } \\
\text { Discharge in each category }\end{array}$} \\
\hline & $\mathrm{A}$ & $\mathrm{B}$ & $\mathrm{C}$ & $\mathrm{D}$ & $\mathrm{E}$ & $\mathrm{A}$ & $\mathrm{B}$ & $\mathrm{C}$ & $\mathrm{D}$ & $\mathrm{E}$ \\
\hline Spinal cord edema & 8 & 0 & 3 & 13 & 0 & 6 & 0 & 5 & 13 & 0 \\
\hline Cord contusion & 10 & 3 & 4 & 0 & 0 & 8 & 3 & 6 & 0 & 0 \\
\hline $\begin{array}{l}\text { Spinal cord injury due to } \\
\text { Extrinsic compression }\end{array}$ & 7 & 0 & 5 & 2 & 0 & 6 & 0 & 6 & 2 & 0 \\
\hline Focus of hemorrhage & 8 & 1 & 0 & 0 & 0 & 7 & 1 & 1 & 0 & 0 \\
\hline Epidural hematoma & 0 & 0 & 1 & 2 & 0 & 0 & 0 & 0 & 3 & 0 \\
\hline Total & 33 & 4 & 13 & 17 & 2 & 27 & 4 & 18 & 18 & 2 \\
\hline
\end{tabular}

Figure I: Graphic comparison of ASIA impairment scale at the time of Admission and Discharge in patients of Spinal Trauma

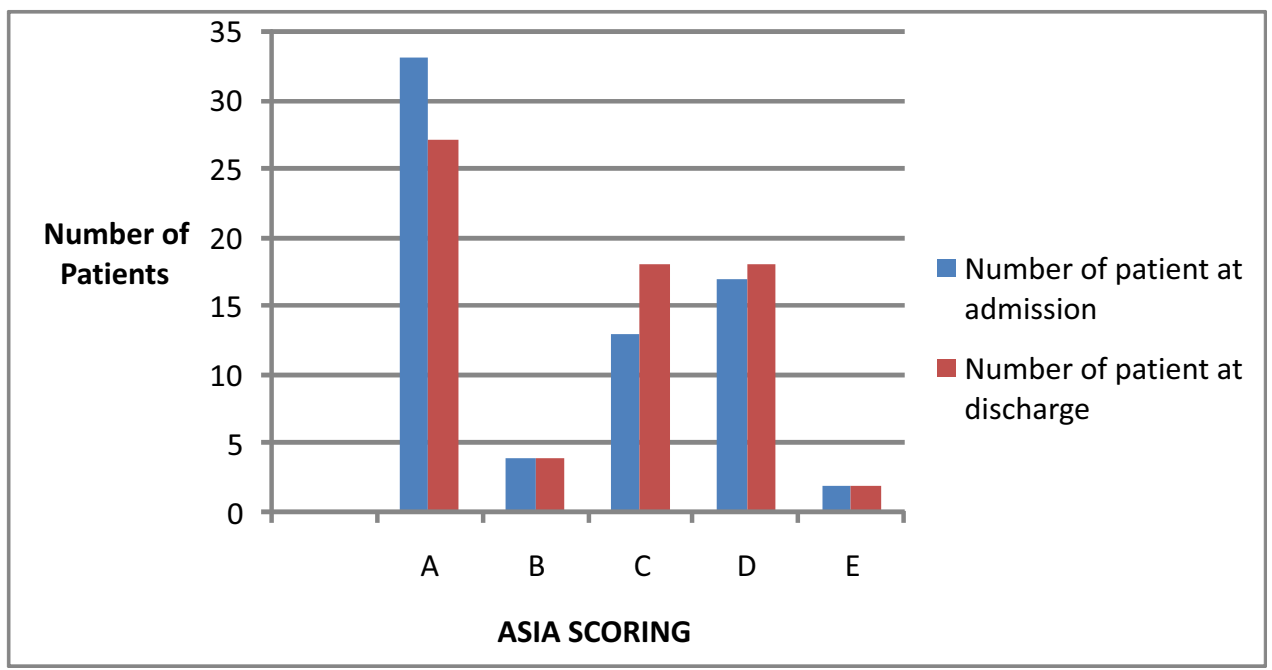

Figure II: MRI showing cord contusion at $\mathrm{C6} / \mathrm{C} 7$ level

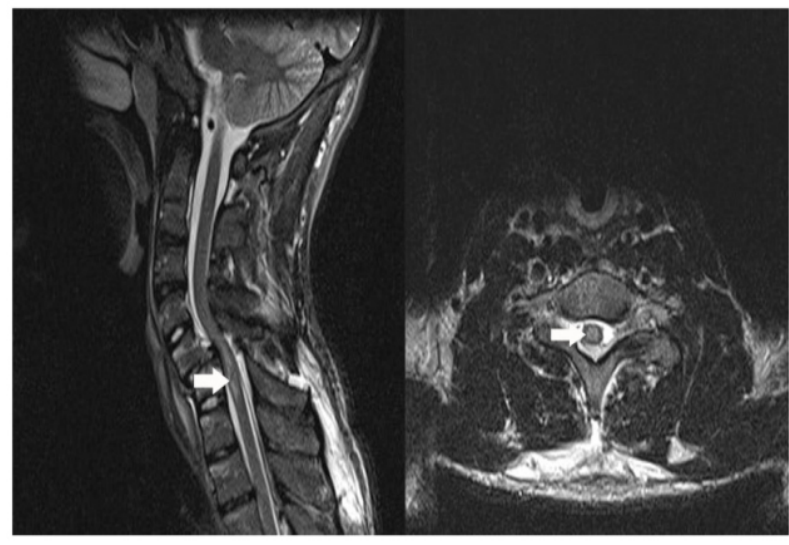

\section{Discussion}

In our study, 7 of 69 patients (10\%) showed improvement and 63 patients showed no improvement while 2 cases had normal spinal cord. Recovery from spinal cord hemorrhage depend upto the extent of the injury. The initial injury of cord in relation with permanent impairment on follow up was described by Boldin et al.10 Their study showed that patients with hemorrhagic injury were more likely to have completed SCI. Similar, as our result that severity of injury have strong association with extent of recovery. Out of 33 patients, with initial ASIA score A, only one third patients $6(18 \%)$ have improved while out of 17 patients with initial ASIA score of D 1 patient, $5.8 \%$ showed 
improvement, in patients with initial ASIA score $\mathrm{C}$, five patients $(38 \%)$ ) showed improvement. It suggests that chance of improvement are less in patients with initial high ASIA scoring. Similar study by Boldin et al, proved that hemorrhage and length of the injured cored effect status of recovery of patient rather severity of injury has inverse proportional relation with recovery of the patient.10 They showed that patients with hemorrhagic presentation were more likely to have completed spinal cord injury (SCI) at the time of follow-up (odds ratio $=2.33,95 \%$ confidence interval, 1.42-3.82). Poor prognostic factors were including long length of cord edema, spinal contusion and high cervical location. Selden NR11 also showed similar results presence of long length of intra-axial hematoma and cord edema, each associated with poor neurological outcome.

Patients with spinal cord edema and cord contusion, two patients showed improvement from initial ASIA category A to category C out of total 24 and 17 patients respectively. Most of the studies proved that cord edema having good result regarding Asia scoring. 12,13,14 Patients present focal single level edema had better initial neurological status than patients with diffuse cord edema. Single level focal edema patients showed two grades improvement in follow up ASIA scoring as compared to patients with diffuse spinal edema. Most of these studies related to the dorsal spinal cord but in our study we conclude similar results for lumbar spine as well.

Spinal cord injury due to extrinsic compression, focus of hemorrhage and epidural hematoma, one patient showed improvement out of total 14, 9 and 3 patients respectively. Series of articles describing disc herniation with injuries to the cervical spine, there was high rate of disc herniation or injury $(36 \%)$ on initial MRI along with higher proportion of posterior ligament complex (PLC) injury as compared to ALL injuries $(64 \%$ vs. $37 \%)$. A study reported that $18 / 134(13 \%)$ of injured had concurrent ligamentous injury but they did not report about the specific ligaments.14 The majority of authors did not state of focal neurological deficit could be attributed to the disc herniation. ${ }^{15-17}$

\section{Conclusion}

Our study showed that the severity of MRI appearance of traumatic spinal cord injuries in acute phase and long-term recovery of motor and sensory functional deficit are closely related. Patients with initial hemorrhagic grade had very poor prognosis, whereas those with focal spinal cord edema had good clinical recovery, as demonstrated by the passage to a higher Frankel class. Magnetic Resonance Imaging having key role in the initial evaluation of unconscious patients who cannot undergo motor and sensory neurological evaluation and it also define the prognosis, which will influence the correct therapeutic choice.

\section{Authors Contribution:}

TA: Conception and revising. OS: Design of work and Drafting. MWA: Interpretation and revising. All the authors gave the final approval for publishing and agreed to be accountable for all aspect of work.

\section{Acknowledgment}

Special thanks for the department of neurosurgery and neurology for their kind cooperation in the collection of data and guiding us in completion of this article.

\section{Conflict of Interest: None \\ Sources of Funding: Self}

\section{References}

1. Chin LS. Spinal Cord Injuries. 2015 [cited 2015]; Availablefrom: http://emedicine.medscape. com/article/793582-overview.

2. TT Roberts, G R leonard,DJ cepela.Classifications In Brief: American Spinal Injury Association (ASIA) Impairment Scale. Clin Orthop Relat Res 2017 May; 475(5): 1499-1504

3. Parizel P, Van der Zijden T, Gaudino S, Spaepen M, Voormolen M, Venstermans C, et al. Trauma of the spine and spinal cord: imaging strategies. Eur Spine J 2010; 19(1):8-17.

4. Provenzale J. MR imaging of spinal trauma. Emerg Radiol 2007 Mar; 13(6):289-97.

5. Sliker CW, Mirvis SE, Shanmuganathan K. Assessing Cervical Spine Stability in Obtunded Blunt Trauma Patients: Review of Medical Literature 1. Radiology 2005; 234(3):733-9.

6. FDA OKs device to help people with some spinal injuries walk [database on the Internet]. Medscape Medical News. 2014 
7. FDA news release. FDA allows marketing of first wearable, motorized device that helps people with certain spinal cord injuries to walk. US Food and Drug Administration; 2015 [cited 2015]; Available from: http://www.fda.gov/NewsEvents/Newsroom/PressAn nouncements/ucm402970.htm.

8. American Spinal Injury Association. International Standards for Neurological Classifications of Spinal Cord Injury. Revised ed. Chicago, Ill: American Spinal Injury Association; 2000: p.1-23.

9. Parashari UC, Khanduri S, Bhadury S, Kohli N, Parihar A, Singh R, et al. Diagnostic and prognostic role of MRI in spinal trauma, its comparison and correlation with clinical profile and neurological outcome, according to ASIA impairment scale. J Craniovertebral Junc Spine. 2011;2(1):17.

10. Boldin C1, Raith J, Fankhauser F, Haunschmid C, Schwantzer G, Schweighofer F.Predicting neurologic recovery in cervical spinal cord injury with postoperative MR imaging. Spine (Phila Pa 1976). 2006 Mar 1; 31(5): 554-9.

11. 11. Selden NR, Quint DJ, Patel N, d'Arcy HS, Papadopoulos SM. Emergency magnetic resonance imaging of cervical spinal cord injuries: clinical correlation and prognosis. Neurosurgery. 1999 Apr; 44(4): 785-92

12. Song KJ, Kim GH, Lee KB. The efficacy of the modified classification system of soft tissue injury in extension injury of the lower cervical spine.Spine (Phila Pa 1976). 2008 Jul 1;33(15):E488-93.
13. Miyanji F, Furlan JC, Aarabi B, Arnold PM, Fehlings MG.Acute cervical traumatic spinal cord injury: MR imaging findings correlated with neurologic outcome-prospective study with 100 consecutive patients. Radiology. 2007 Jun; 243(3): 820-7.

14. Taneichi H. Suda K. Kajino T. Kaneda K. Traumatically induced vertebral artery occlusion associated with cervical spine injuries: Prospective study using magnetic resonance angiography. Spine (Phila Pa 1976) 2005; 30: 1955-1962

15. Goradia D, Linnau KF, Cohen WA, Mirza S, Hallam DK, Blackmore C. Correlation of MR imaging findings with intraoperative findings after cervical spine trauma. AJNR Am J Neuroradiol. 2007 Feb; 28(2): 209-15.

16. Song KJ, Kim GH, Lee KB. The efficacy of the modified classification system of soft tissue injury in extension injury of the lower cervical spine. Spine (Phila Pa 1976). 2008 Jul 1;33(15):E488-93.

17. Labattaglia MP, Cameron PA, Santamaria M, Varma D, Thomson K, Bailey M, Kossmann T. Clinical outcomes of magnetic resonance imaging in blunt cervical trauma.Emerg Med Australas. 2007 Jun; 19(3): 253-61. 